\title{
Über Risiken und Nebenwirkung fachfremder Kontrolle
}

Krankenkassen und Ärztenetzwerke gehen davon aus, Managed Care senke die Kosten bei gleichbleibender oder gar besserer Qualität. Der Verein Ethik und Medizin Schweiz hat den Ökonomen Mathias Binswanger mit der Überprüfung dieser Behauptung beauftragt. Die hier vorgestellten Ergebnisse veranlassen den Autor, ein System zur ärztlichen Selbstkontrolle zu fordern, z. B. ein Dysfunction Board als Institution der FMH mit Sanktionsrecht.

Korrespondenz: Dr. med. Michel Romanens Präsident Verein Ethik und Medizin Ziegelfeldstrasse 1 CH-4600 Olten

info[at]kardiolab.ch

\section{Vertauschte Rollen}

Stellen Sie sich folgende Situation vor: Sie sind Hausbesitzer und haben eine Gebäudeversicherung abgeschlossen. Nun geht diese ohne Ihr Wissen Vereinbarungen mit den Handwerkern ein, die im Schadensfall Ihr Haus reparieren. Diese Vereinbarungen sehen vor, dass die Handwerker im Schadensfall einen Teil des Risikos für die Reparatur tragen und die Gebäudeversicherung im Gegenzug Funktionen übernimmt, die sonst der Bauherr innehat. Das klingt abstrus? Ganz so viel anders sieht das Managed-Care-Modell aber gar nicht aus. Die ökonomische Existenzberechtigung einer Versicherung ist es, das Schadensrisiko zu tragen. Bei einer Krankenversicherung sind das die Behandlungskosten. Wenn, wie bei Managed Care, diese Kosten mit dem Netzwerk in einem Budget festgehalten werden, für welches das Netzwerk in einem vereinbarten Bonus-Malus-System eine Mitverantwortung übernimmt, dann übernimmt jeder teilweise Aufgaben des anderen. Der Leistungserbringer kann wohl das Schadensrisiko nicht beeinflussen, die «Reparaturkosten» muss er aber im vereinbarten Budget halten. Die Kasse ihrerseits nimmt auf den Indikationsentscheid und auf die Behandlung Einfluss, allein damit, dass ein solches Budget festgelegt wird. Die Behauptung der Krankenkassen, mit diesem Modell liessen sich bei gleichbleibender oder gar höherer Qualität Kosten sparen, ist einerseits nie bewiesen worden [1], andererseits widerspricht sie auch dem, was wir über Arbeitsteilung gelernt haben. Wieso sollte denn Geld zu sparen sein, indem einer einen Teil der Arbeit des anderen macht, für den er fachlich nicht qualifiziert ist?

\section{Welcher Anreiz ist der richtige?}

Natürlich hinkt unser Vergleich. Der Gesundheitsmarkt ist kein freier Markt. Der Marktmechanismus wird hier von der Nachfrageseite her ausser Kraft gesetzt. Die Krankenkasse ist obligatorisch, der Kunde zahlt bezogene Dienstleistungen nicht direkt, son-

\section{Risques et effets secondaires des contrôles par des non- professionnels}

Le présent article aborde la problématique de fond relative au contrôle des prestations médicales par des non-professionnels et montre où il faudrait aller, à savoir vers un contrôle des disfonctionnements médicaux par les médecins eux-mêmes, comme c'est d'ailleurs le cas dans d'autres secteurs professionnels. L'Association Ethique et Médecine fait ici une proposition concrète et invite les lecteurs à œuvrer de manière à parvenir ensemble à une meilleure situation.

dern indirekt mit der Versicherungsprämie (sogenanntes third-party payment). In einem solchen System kommt es naturgemäss sowohl zu einer Überproduktion als auch zu einem Überkonsum. Welchen Grund hätte der Kunde (Patient) denn schon, nicht die beste Behandlung zu wählen, ohne Rücksicht auf die Kosten? Und wieso sollte der Anbieter (Arzt) bei zwei gleich wirksamen Behandlungen nicht jene wählen, die für ihn am lukrativsten ist? Hier braucht es regulierende Kräfte, denn der Anreiz ist im Prinzip falsch gesetzt: Der Arzt verdient umso mehr, je mehr er behandelt. Ärztenetzwerke mit Budgetmitverantwortung drehen diesen Anreiz um: Je weniger der Arzt behandelt, desto mehr verdient er.

\section{Gutachten schafft Klarheit}

Der Verein Ethik und Medizin Schweiz wollte wissen, ob dieser Anreiz nicht nur neu, sondern auch besser ist. Die Krankenkassen und die Betreiber von Ärztenetzwerken gehen davon aus, Managed Care 
sei nicht nur kosteneffektiv (man kann die Kosten senken), sondern auch kosteneffizient (man kann die Kosten senken und gleichzeitig die Qualität beibehalten oder gar verbessern). Um der Frage nachzugehen, ob dem tatsächlich so ist, haben wir den Ökonomen Prof. Mathias Binswanger von der Universität St. Gallen beauftragt, ein Gutachten zu erstellen, das die finanziellen Anreizwirkungen der Budgetmitverantwortung untersucht [1]. Dieses Gutachten kann auf www.vems.ch/geheimvert raege heruntergeladen werden. Die von Prof. Binswanger durchgeführte Literaturübersicht zeigt, dass es keine Studien gibt, welche die Kosteneffizienz der Budgetmitverantwortung nachweisen. Es ist also nicht klar, ob die im Rahmen von Managed-CareNetzwerken realisierten Einsparungen dank der Vermeidung überflüssiger oder aufgrund der Vermeidung notwendiger Leistungen realisiert wurden [2]. Anhand von Modellrechnungen konnte Prof. Binswanger hingegen feststellen, dass mit den existierenden Verträgen Anreize geschaffen werden, die den Hausarzt dafür belohnen, Weiterweisungen an andere Institutionen oder Spezialisten möglichst lange hinauszuzögern oder ganz zu vermeiden. Gleichzeitig kann er seine eigenen Leistungen aus-

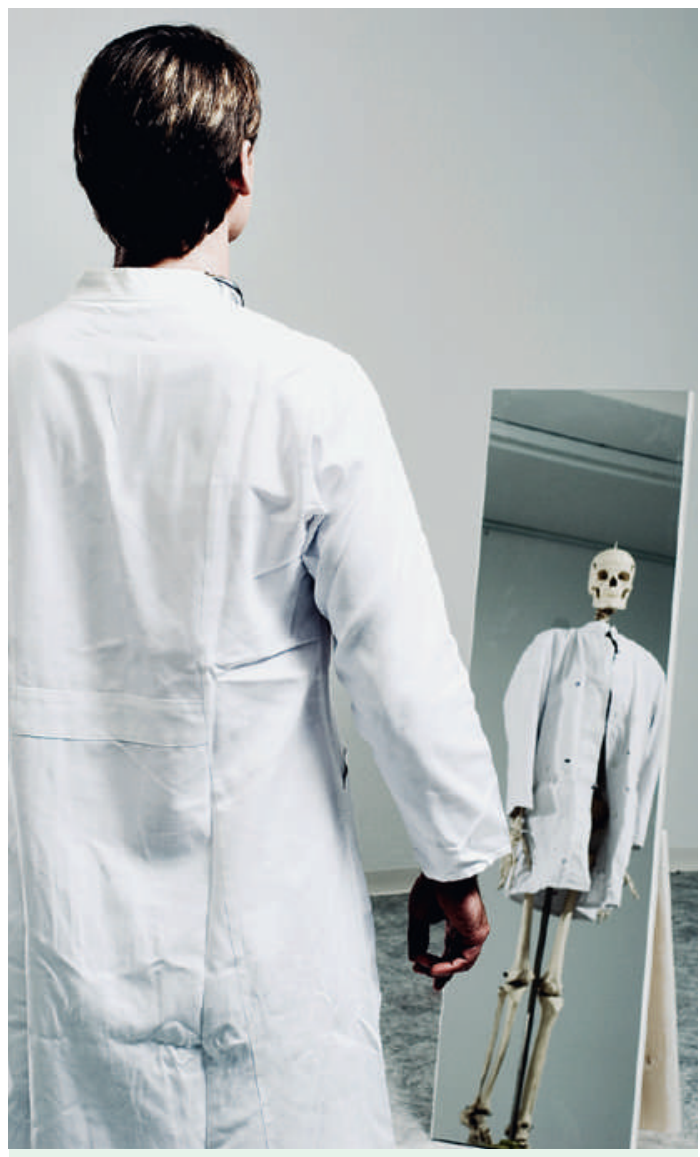

Wie bedrohlich ist die fachfremde Kontrolle der Ärztinnen und Ärzte für die Sicherheit der Patientinnen und Patienten? (Bild: Nanduu/photocase.com) weiten, wenn er über die gesamte Kette der gesetzlichen Pflichtleistungen die Kosten unter dem vereinbarten Einsparungsziel hält.

\section{Geheimverträge regeln das Entscheidende}

Dies bedeutet: Um Managed Care überhaupt beurteilen zu können, müssen die darin gesetzten Anreize sorgfältig geprüft werden. Diese sind: Abgrenzung des Netzwerk-Budgets, Berechnung der Erfolgs- bzw. Verlustbeteiligung, Verwendung der Überschüsse durch das Netzwerk, Umgang mit Grossrisiken und Qualitätskontrolle. Genau diese Bestimmungen werden heute aber in Geheimverträgen zwischen den Kassen und den Netzwerken vereinbart. Da sowohl die monetären Vereinbarungen als auch die Qualitätskontrolle in bestehenden Netzwerken öffentlich nicht zugänglich und in der Hand der Kassen sind, besteht die Gefahr, dass Qualität so gemessen wird, dass sie die einseitig wirtschaftlichen Vorgaben der Kasse erfüllt, und dass Anreize so gesetzt werden, dass sie zu Handlungen führen, die nicht zum Wohle des Patienten sind.

\section{Selbstkontrolle ist unabdingbar}

Das Gutachten von Mathias Binswanger zeigt also, dass ökonomische Anreize, entgegen den Behauptungen der Krankenkassen, nicht geeignet sind, die Qualität und die Wirtschaftlichkeit zu verbessern. Wie die Wirtschaftlichkeitsverfahren der santésuisse und die Fallpauschalen, so läuft auch die Budgetmitverantwortung darauf hinaus, dass Krankenkassen Kontrollfunktionen übernehmen, die nur medizinische Fachpersonen wahrnehmen können. Das ist ein Unikum. Während die Kontrolle in anderen Branchen zumeist durch Fachleute und -verbände selbst geleistet wird, sieht sich der Arzt heute zunehmend von fachfremden Personen und Organisationen kontrolliert. Wieso? Können wir uns nicht selbst kontrollieren? Oder wollen wir nicht? Die Konzepte, wie im Gesundheitswesen wirklich und nachhaltig gespart werden kann, sind uns Ärzten doch längst geläufig: Je früher eine Krankheit erkannt wird, desto kostengünstiger ist ihre Behandlung. Dass diese Behandlung gleichzeitig für den Patienten angenehmer ist, zeigt, dass wir Ärzte, wenn wir Wirtschaftlichkeit richtig verstehen, nicht gegen, sondern für das Wohl unserer Patienten arbeiten. Der Patient wünscht eine möglichst schmerzfreie Behandlung, ein Arzt, der ihm unnötiges Leiden verursacht - sei es durch einen ökonomischen Anreiz zur Überversorgung oder durch einen solchen zur Unterversorgung - ist dysfunktional und gehört revalidiert. Und zwar nicht durch fachfremde Gremien oder Krankenkassen, sondern durch uns Ärzte selbst. Wenn wir uns heute einer zügellosen Kontroll- und Qualitätslabelindustrie ausgesetzt sehen, deren Nutzen nicht erwiesen, deren Kosten aber bekannt sind, dann müssen wir Ärzte uns doch auch fragen, weshalb man uns denn so sehr misstraut. Die Antwort ist unangenehm: Weil wir es versäumt haben, in 
unserer Branche entsprechende Systeme zur Selbstkontrolle zu installieren.

\section{Kontrolle von aussen ist schädlich}

Die Folge ist eine Kontrolle von aussen, die nachweislichen Schaden anrichtet. Die Versuche, auf den Entscheid und auf die Behandlung des Arztes mittels wirtschaftlicher Bonus- und Buss-Systeme Einfluss zu nehmen, sind so vielfältig wie fragwürdig. Die Wirtschaftlichkeitsverfahren der santésuisse, Budgetmitverantwortung und Fallpauschalen zementieren die Annahme, der Arzt gehöre wirtschaftlich überwacht, weil er sich sonst auf Kosten seines Patienten bereichere. Dabei weiss doch jeder Arzt im Grunde sehr genau, wie falsche Indikationsentscheide erfassbar sind. Wieso also akzeptieren wir diese einseitig auf Kosten ausgerichtete Kontrolle von aussen, die ärztliche Dysfunktion nicht erfasst, sondern unter Umständen sogar fördert? Am Beispiel der Wirtschaftlichkeitsverfahren lässt sich dies gut illustrieren: Durch die einseitig auf Wirtschaftlichkeit ausgerichtete Beurteilung des Arztes wird Dysfunktion nicht erkannt, sondern im Gegenteil verursacht, indem der Arzt einem ökonomischen Druck ausgesetzt wird, der ihn zwingt, nicht mehr das Wohl seines Patienten in den Mittelpunkt seiner Arbeit zu stellen, sondern sein eigenes wirtschaftliches Überleben. Wir haben also eine durch ein Instrument zur Überwachung ärztlicher Dysfunktion verursachte ärztliche Dysfunktion. Und sie richtet auch wirtschaftlichen Schaden an, indem in der Prävention Versäumnisse begangen werden, die teure Behandlungen nach sich ziehen.

\section{Systematische Erfassung ärztlicher Dysfunktion}

Die Bioethik in der Medizin hat lange einen Mangel an Ressourcen postuliert. Kostenkontroll-Instrumente wie Wirtschaftlichkeitsverfahren, QALY oder Budgetmitverantwortung werden mit dem Schlagwort der «distributiven Gerechtigkeit» gerade auch in der Schweiz als einzig gangbarer Weg der Kostenkontrolle durchgesetzt. In den USA kommt man jedoch weg von der unbewiesenen Hypothese des Ressourcenmangels und bewegt sich hin zur Bioethik der «Waste Avoidance» [4]. Diese Sichtweise entspricht auch den expliziten Äusserungen des BAG, wonach noch Raum besteht für Rationalisierungen. Der Knackpunkt: Rationalisierung muss auf höchstem professionellem Niveau erfolgen. Dieses Ziel kann ohne Selbstkontrollen unter Verwendung von Appropriateness Criteria nicht erreicht werden. Was in der Schweiz fehlt, sind eine Systematik ärztlicher Dysfunktion einerseits und Instanzen zur Erfassung dieser Dysfunktionen andererseits.

\section{Ein konstruktiver Vorschlag}

Der Verein Ethik und Medizin Schweiz hat zu diesem Zweck das Projekt Dysfunction Assessment ins Leben gerufen. Auf www.vems.ch/dysfunction-assessment haben wir eine Auflistung möglicher ärztlicher Dysfunktionen aufgeschaltet. Diese Tabelle kann von interessierten Kolleginnen und Kollegen bearbeitet und kommentiert werden. Unser Vorschlag, wie ein System zur Erfassung dieser Dysfunktionen aussehen könnte, findet sich im gleichen Dokument. Erster Pfeiler: ein Dysfunction Board als Institution der FMH mit Sanktionsrecht. Auffällige Ärzte sollen dem Board gemeldet werden, durch Patientinnen und Patienten, aber auch durch die Ärzte selbst. Das Board trifft dann aufgrund des institutionalisierten Vorgehens entsprechende Abklärungen. Zweiter Pfeiler: ein Peer Review System. Es braucht bei der Indikationsstellung für Abklärungen, Behandlungen und Eingriffe mit hoher Kostenfolge ein externes und freiwilliges System, in dem beispielsweise Spitäler untereinander die Richtigkeit von Entscheiden anhand zufälliger Reviews ( $A u$ dits) kontrollieren. Im US-Staat Maryland hat ein Team um Dr. Sam Goldberg, Dr. Roger Leonard und Dr. Marc Mugmon bei der Legislative und den Aufsichtsbehörden hartnäckig für ein System externer peer reviewers geworben, um so stichprobenartige externe Beurteilungen aller Koronareingriffe im Staat Maryland zu erhalten [5]. Die Notwendigkeit eines solchen Systems ist also auch anderswo erkannt. Dritter Pfeiler: eine qualitativ hochstehende Institutionalisierung der second opinion. Diese Arbeit können nur finanziell und institutionell von Kassen, Spitälern oder sonstigen Interessenvertretern unabhängige Fachpersonen leisten, die hierfür speziell bezeichnet werden, z. B. von Fachgesellschaften.

\section{Nehmen wir es in die Hand}

Wir Ärzte müssen uns heute entscheiden: Wollen wir uns weiterhin der naiven Hoffnung hingeben, Institutionen wie santésuisse, die Behörden, die privaten Quality-Assessments der Industrie, die Ombudsstellen der Kassen oder Patientenorganisationen verfügten über die Unabhängigkeit und die Professionalität, Standards zur Erfassung ärztlicher Dysfunktion zu setzen? Oder wollen wir diese Systeme nicht lieber selber schaffen? Denn wer, wenn nicht wir Ärzte selbst, sollte in der Lage sein, unsere Arbeit zu beurteilen? Ihre Mitarbeit ist gefragt!

\section{Referenzen}

1 http://physicianprofiling.ch/InfoGutachtenBinswanger052012.pdf

2 http://physicianprofiling.ch/RationingDeterminantsEuropeAndSwitzerlandHurst2006.pdf

3 http://physicianprofiling.ch/InfoGGeheimvert raege052012.pdf

4 http://physicianprofiling.ch/RationingVersusWaste AvoidanceNEJM052012.pdf

5 http://www.marylandacc.org/presidents-corner/ presidents-corner.html 